九州大学学術情報リポジトリ

Kyushu University Institutional Repository

膵癌に対する重粒子線治療後の上部消化管潰瘍発生 における線量解析

篠藤，誠

http://hdl. hand le. net/2324/2236334

出版情報：Kyushu University，2018，博士（医学），論文博士 バージョン：

権利関係 : 
Particle therapy of pancreas

\title{
Dosimetric analysis of upper gastrointestinal ulcer after carbon-ion radiotherapy for pancreatic cancer
}

\author{
Makoto Shinoto $^{\mathrm{a}, \mathrm{b}, *}$, Yoshiyuki Shioyama ${ }^{\mathrm{a}}$, Akira Matsunobu ${ }^{\mathrm{a}}$, Kei Okamoto ${ }^{\mathrm{a}}$, Hiroaki Suefuji ${ }^{\mathrm{a}}$, \\ Shingo Toyama ${ }^{\mathrm{a}}$, Hiroshi Honda ${ }^{\mathrm{b}}$, Sho Kudo ${ }^{\mathrm{a}}$ \\ ${ }^{a}$ Ion Beam Therapy Center, SAGA HIMAT Foundation, Tosu; and ${ }^{\mathrm{b}}$ Department of Clinical Radiology, Graduate School of Medical Sciences, Kyushu University, Fukuoka, Japan
}

\section{A R T I C L E I N F O}

\section{Article history:}

Received 14 March 2016

Received in revised form 12 April 2016

Accepted 28 April 2016

Available online 10 May 2016

\section{Keywords:}

Pancreatic cance

Carbon-ion radiotherapy

Dose volume histogram

Gastrointestinal ulcer

\begin{abstract}
A B S T R A C T
Purpose: The aim of this study was to clarify the incidence, clinical risk factors, and dose-volume relationship of upper gastrointestinal (GI) ulcer after carbon-ion radiotherapy (C-ion RT) for pancreatic cancer.

Materials and methods: Fifty-eight pancreatic cancer patients were treated with C-ion RT from April 2014 to December 2015. The total dose was $55.2 \mathrm{~Gy}$ (RBE) in 12 fractions. $\mathrm{D}_{2 \mathrm{~cm} 3}$ of GI tracts were restricted under $46 \mathrm{~Gy}$ (RBE); RBE-weighted absorbed dose. The association between dosimetric parameters $\left(V_{10-50}, D_{\max }, D_{1 \mathrm{~cm} 3}, D_{2 \mathrm{~cm} 3}\right)$ and GI ulcer was examined using Spearman's correlation. The incidence of GI ulcer was compared between the two groups divided by the cutoff value.

Results: Twelve patients (21\%) experienced gastric ulcer including only one (2\%) grade 3 ulcer. There was no grade $4 / 5$ toxicity or duodenal ulcer. $\mathrm{V}_{10-30}$ was significantly associated with gastric ulcer. The 1 -year estimated risk of gastric ulcer for the determined cutoff values were $51 \% \mathrm{vs} .10 \%\left(\mathrm{~V}_{10}, \geqslant 102 \mathrm{~cm}^{3}\right.$ or less) $42 \%$ vs. $9 \%\left(\mathrm{~V}_{20}, \geqslant 24 \mathrm{~cm}^{3}\right.$ or less), $34 \%$ vs. $4 \%\left(\mathrm{~V}_{30}, \geqslant 6 \mathrm{~cm}^{3}\right.$ or less).

Conclusions: The incidence of GI ulcer after C-ion RT was very low with the dose constraint of $\mathrm{D}_{2 \mathrm{~cm} 3}$ $<46 \mathrm{~Gy}$ (RBE). To further minimize the risk of GI ulcer, $\mathrm{V}_{10-30}$ should also be reduced.
\end{abstract}

(C) 2016 Elsevier Ireland Ltd. All rights reserved. Radiotherapy and Oncology 120 (2016) 140-144
The role of radiotherapy for pancreatic cancer is controversial. Two recent randomized studies to evaluate the role of chemoradiotherapy (CRT) compared with chemotherapy alone in locally advanced pancreatic cancer showed conflicting results [1,2]. Preliminary data from the LAP 07 trial revealed no clear benefit from consolidative CRT following chemotherapy [3]. This is partly because pancreatic cancer is radioresistant; meanwhile, the sensitivity of the organs in the upper abdomen has limited radiation doses to levels that are ineffective against pancreatic cancer [4].

A dose escalation trial of carbon-ion radiotherapy (C-ion RT) with concurrent gemcitabine for locally advanced pancreatic cancer was previously conducted in National Institute of Radiological Sciences in Japan and achieved improved survival, with a median survival time of 23.9 months and a 2 -year survival rate of $48 \%$ [5]. Carbon-ion beams offer improved dose distribution and provide greater biological effectiveness than photons or protons [6,7]. C-ion RT can administer a high-intensity dose to the target, which exceeds the tolerance dose of normal tissue, especially the gastrointestinal (GI) tract. In a previous C-ion RT dose escalation

\footnotetext{
* Corresponding author at: Ion Beam Therapy Center, SAGA HIMAT Foundation, 3049 Harakoga-machi, Tosu, Saga 841-0071, Japan.

E-mail address: shinoto@saga-himat.jp (M. Shinoto).
}

trial, the incidence of GI toxicity was very low, so the maximum tolerated dose could not be identified [5]. Only one patient (1\%) experienced late grade 3 gastric ulcer, and about half of the patients who were prescribed $55.2 \mathrm{~Gy}$ (RBE), which is the relative biologic effectiveness (RBE)-weighted absorbed dose defined in ICRU report 78 [8], experienced acute grade 1 or 2 GI ulcers. Based on these data, the maximal absolute dose that covered $2 \mathrm{~cm}^{3}$ $\left(D_{2 \mathrm{~cm} 3}\right)$ of the GI tract was indicated to be restricted under $46 \mathrm{~Gy}$ (RBE). The identification of reliable predictors for GI ulcer will be important for future dose escalation studies. However, no prior study to our knowledge has investigated the dose-toxicity relationship for the treatment of pancreatic cancer with C-ion RT.

Our aim in this study was to verify the assurance of the dose constraint of $\mathrm{D}_{2 \mathrm{~cm} 3}<46 \mathrm{~Gy}$ (RBE), and to explore the predictive factors of risk for upper GI ulcer from the dose-volume histogram $(\mathrm{DVH})$ of the organs at risk in pancreatic cancer patients treated with C-ion RT.

\section{Materials and methods}

\section{Patients and treatment}

We retrospectively analyzed 58 consecutive pancreatic cancer patients who were treated with curative intent by C-ion RT at 
our institution from April 2014 to December 2015. Fifty-three patients were treated with concurrent chemotherapy, namely gemcitabine or S-1 based chemotherapy. Forty-five patients underwent chemotherapy before the initiation of C-ion RT. Most patients were recommended to undergo adjuvant chemotherapy. In all patients, gastroduodenal-protective drugs were administered prophylactically at the beginning of C-ion RT.

\section{Carbon-ion radiotherapy}

The details of the C-ion RT technique were approximately same as those reported previously [5]. In our institution, a set of 2-mmthick non-contrast-enhanced computed tomography (CT) images was taken under respiratory gating for treatment planning purposes. Three-dimensional treatment planning of C-ion RT was performed using the XiO-N (ELEKTA, Stockholm, Kingdom of Sweden and Mitsubishi Electric, Tokyo, Japan) software program.

The gross tumor volume (GTV) was determined mainly by contrast-enhanced dynamic CT. Magnetic resonance imaging or 18 fluoro-2-deoxyglucose positron emission tomography was also taken into account. The clinical target volume (CTV) was defined as a GTV with a 5-mm margin and a locoregional elective nodal and neuroplexus region. The locoregional elective nodal regions, which are classified as N2 stations according to the General Rules for Cancer of the Pancreas published by the Japan Pancreas Society [9], included the celiac, superior mesenteric, peri-pancreatic, portal, and para-aortic region for pancreatic head cancer and the splenic region for pancreatic body and tail cancer. The planning target volume (PTV) was defined as the clinical target volume with a $5-\mathrm{mm}$ margin for possible positioning errors, respecting anatomical boundaries such as the stomach, duodenum, and small intestine. In cases in which the tumor was located close to critical organs, the margin was reduced accordingly.

The planned total dose was $55.2 \mathrm{~Gy}$ (RBE) in 12 fractions. The RBE value was estimated to be 3.0 at the distal part of the spread-out Bragg peak and the biological model was reported in reference [6]. The $D_{2 \mathrm{~cm} 3}$ of the GI tracts was restricted to under $46 \mathrm{~Gy}$ (RBE). At least $90 \%$ of the PTV received at least $95 \%$ of the prescribed dose. The beam arrangements were a four-field plan.

\section{Dosimetric analysis and evaluation of upper gastrointestinal ulcer}

The external surfaces of the critical organs including stomach, duodenum and small intestine were contoured on each axial slice of the planning CT images. A dose-volume histogram (DVH) was calculated using a 3-dimensional planning computer (XiO-N). The following dosimetric parameters were generated from the DVH: the maximal absolute dose, covering $1 \mathrm{~cm}^{3}$ of the organ $\left(D_{1 \mathrm{~cm} 3}\right)$, $\mathrm{D}_{2 \mathrm{~cm} 3}$, and the absolute volume of the organ receiving more than a threshold dose, with a dose of 10-50 Gy (RBE) in increments of $10 \mathrm{~Gy}$ (RBE) $\left(\mathrm{V}_{10}-\mathrm{V}_{50}\right)$. Additionally, the absolute PTV was also acquired.

GI ulcer was evaluated according to the Common Terminology Criteria of Adverse Events, version 4.0. Acute toxicity was defined as toxicity that occurred within 3 months from the start of C-ion RT. Late toxicity was defined as toxicity that occurred after 3 months. In all patients, upper GI endoscopy was performed before and 1 month after C-ion RT. Subsequent endoscopic explorations were performed at the time of any sign of upper GI pain or discomfort or anemia, or bloody stool, or at least every six months when there were no signs or symptoms.

\section{Statistical analysis}

The study analyzed clinical and dosimetric parameters affecting the development of GI ulcer. Gender, age, chemotherapy, tumor size, tumor location, clinical stage, PTV, and chemotherapy were considered binary variables. Furthermore, Spearman's correlation was used to examine the strength of association between $\mathrm{V}_{10}-\mathrm{V}_{50}, \mathrm{D}_{\max }, \mathrm{D}_{1 \mathrm{~cm} 3}$, and $\mathrm{D}_{2 \mathrm{~cm} 3}$ of the GI ulcers. As we describe below, GI ulcers appeared only in the stomach, so that the DVH parameter of the stomach was analyzed in this study. A receiver operating characteristic (ROC) curve was also generated to assess the predictability of dosimetric parameters related to GI ulcer and to determine the optimal cutoff value for each dosimetric parameter. Each dosimetric parameter was divided into two groups using the optimal cutoff value obtained from ROC analysis, and the estimated incidence of GI ulcer was compared between the two groups with the log-rank test. Statistical significance was defined as a $p$ value $<0.05$. Analyses were performed with the JMP 8.0 software (SAS Institute, Inc., Cary, NC).

\section{Results}

\section{Patient characteristics}

The median follow-up time was 9.3 months (range, 3.1-21.3). One-year overall survival was $84 \%$. At the time of analysis, 51 patients $(88 \%)$ were alive. The patient characteristics and treatment details are summarized in Table 1.

\section{Incidence of upper GI ulcer}

Among the 58 patients, gastric ulcers were observed in 12 patients (21\%), and only one patient (5\%) experienced grade 3 ulcer at 1.1 month from the $\mathrm{C}$-ion RT. She was administered a one-time transfusion because of anemia, but active bleeding was not observed. Grade 2 ulcer was observed in 9 patients and grade 1 ulcer in 2 patients. These grade $1 / 2$ ulcers were relieved with proton pump inhibitor and rest without any intensive endoscopic or surgical treatment. Ten (83\%) of 12 ulcers occurred within the acute phase, and the median time of ulcer occurrence was 2.0 months (range, 1.1-8.1). The estimated cumulative incidence

Table 1

Patient characteristics.

\begin{tabular}{ll}
\hline Characteristic & Value \\
\hline Patients $(n)$ & 58 \\
Age (y) & $66(42-92)$ \\
Median (range) & \\
Gender & 29 \\
Male & 29 \\
Female & \\
Clinical stage (UICC 7th) & 8 \\
IIA & 3 \\
IIB & 43 \\
III & 4 \\
IV & \\
Tumor location & 24 \\
Head & 34 \\
Body/tail & \\
Tumor size (mm) & $35(15-70)$ \\
Median (range) & \\
PTV (cm ${ }^{3}$ ) & 197 (83-412) \\
Median (range) & \\
Chemotherapy & 45 \\
Induction chemotherapy & 53 \\
Concurrent chemotherapy & 27 \\
Gemcitabine & 22 \\
S-1 & 4 \\
Gemcitabine and S-1 & \\
\hline
\end{tabular}

Abbreviation: PTV = planning target volume. 


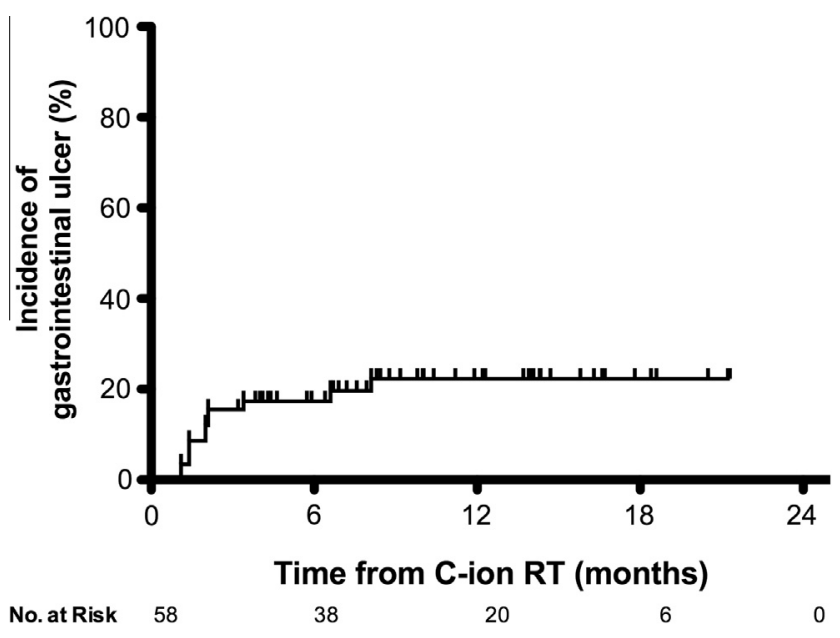

Fig. 1. Cumulative incidence of GI ulcer.

of gastric ulcer is shown in Fig. 1. Most of those were located at the lesser curvature or the posterior wall of the lower stomach regardless of the tumor location. There was no duodenal or small intestine ulcer and bleeding or obstruction. The correlations between ulcer and clinical factors are listed in Table 2.

\section{Dose-volume relationship}

In all patients, $\mathrm{D}_{2 \mathrm{~cm} 3}$ of $\mathrm{GI}$ tracts was restricted under $46 \mathrm{~Gy}$ (RBE). The mean dose of $\mathrm{D}_{2 \mathrm{~cm} 3}$ was $38.0 \mathrm{~Gy}$ (RBE) (range, 18.6-45.4). DVH values of stomachs in all patients are shown in Fig. 2. There were significant correlations between the development of ulcer and $\mathrm{V}_{10}, \mathrm{~V}_{20}$, and $\mathrm{V}_{30}$. By contrast, other dosimetric parameters $\left(\mathrm{V}_{40}, \mathrm{~V}_{50}, \mathrm{D}_{2 \mathrm{~cm} 3}, \mathrm{D}_{1 \mathrm{~cm} 3}, \mathrm{D}_{\max }\right)$ did not significantly correlate with development of ulcer (Table 3). As shown in Table 4, the actual incidence of ulcer when a division was made into two groups with the cutoff value determined by ROC analysis was

Table 2

Correlation between gastrointestinal ulcer and clinical factors.

\begin{tabular}{|c|c|c|c|c|}
\hline Factors & $n$ & GI ulcers & Cumulative 1 -year incidence & $p$ \\
\hline \multicolumn{5}{|l|}{ Age (y) } \\
\hline$\geqslant 66$ & 30 & $8(27 \%)$ & $29 \%$ & \multirow[t]{2}{*}{0.32} \\
\hline$<66$ & 28 & $4(14 \%)$ & $15 \%$ & \\
\hline \multicolumn{5}{|l|}{ Gender } \\
\hline Male & 29 & $8(28 \%)$ & $30 \%$ & \multirow[t]{2}{*}{0.22} \\
\hline Female & 29 & $4(14 \%)$ & $15 \%$ & \\
\hline \multicolumn{5}{|l|}{ Clinical stage } \\
\hline IIA-IIB & 11 & $3(27 \%)$ & $30 \%$ & \multirow[t]{2}{*}{0.62} \\
\hline III-IV & 47 & $9(19 \%)$ & $20 \%$ & \\
\hline \multicolumn{5}{|l|}{ Tumor location } \\
\hline Head & 24 & $4(17 \%)$ & $17 \%$ & \multirow[t]{2}{*}{0.55} \\
\hline Body/tail & 34 & $8(33 \%)$ & $26 \%$ & \\
\hline \multicolumn{5}{|c|}{ Tumor size (mm) } \\
\hline$\geqslant 35$ & 32 & $7(22 \%)$ & $23 \%$ & \multirow[t]{2}{*}{0.74} \\
\hline$<35$ & 26 & $5(19 \%)$ & $20 \%$ & \\
\hline \multicolumn{5}{|l|}{ PTV $\left(\mathrm{cm}^{3}\right)$} \\
\hline$\geqslant 197$ & 28 & $8(29 \%)$ & $30 \%$ & \multirow[t]{2}{*}{0.17} \\
\hline$<197$ & 30 & $4(13 \%)$ & $15 \%$ & \\
\hline \multicolumn{5}{|c|}{ Induction chemotherapy } \\
\hline Yes & 45 & $8(18 \%)$ & $18 \%$ & \multirow[t]{2}{*}{0.26} \\
\hline No & 13 & $4(31 \%)$ & $38 \%$ & \\
\hline \multicolumn{5}{|c|}{ Concurrent chemotherapy } \\
\hline Gemcitabine & 27 & $7(26 \%)$ & $27 \%$ & \multirow[t]{2}{*}{0.50} \\
\hline S-1 & 22 & $5(23 \%)$ & $26 \%$ & \\
\hline
\end{tabular}

Abbreviation: PTV = planning target volume.

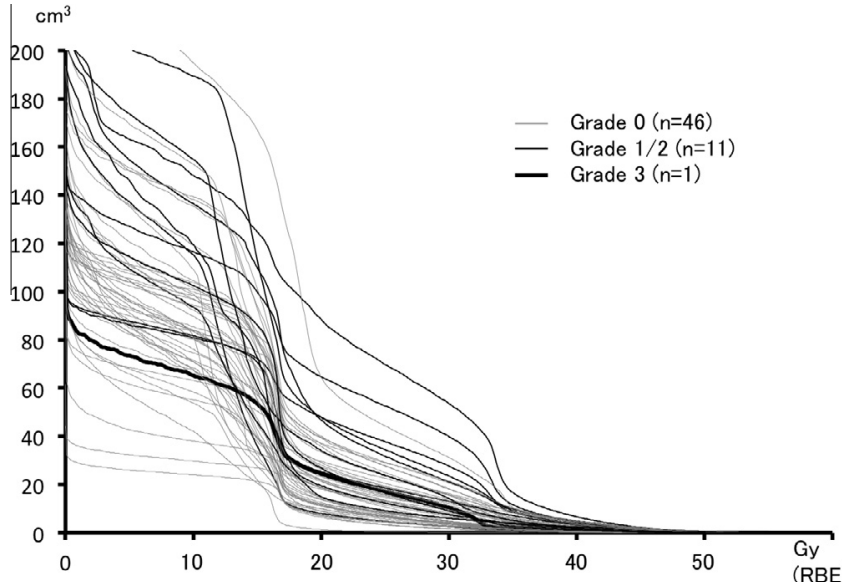

Fig. 2. Dose-volume histogram of stomach for all patients according to gastric ulcer grade (grade 0 vs. $1 / 2$ vs. 3 ). Ulcer cases represented a relatively high dose volume; however, the grade 3 case did not necessarily represent the highest dose volume.

Table 3

Correlation between gastrointestinal ulcer and dosimetric parameters.

\begin{tabular}{|c|c|c|c|c|}
\hline \multirow[t]{2}{*}{ Parameter } & \multicolumn{2}{|l|}{ Mean } & \multirow[t]{2}{*}{ Coefficient } & \multirow[t]{2}{*}{$p$} \\
\hline & Grade 0 & Grade1-3 & & \\
\hline $\mathrm{V}_{10}\left(\mathrm{~cm}^{3}\right)$ & 86.95 & 116.97 & 0.34 & 0.008 \\
\hline $\mathrm{V}_{20}\left(\mathrm{~cm}^{3}\right)$ & 20.39 & 37.38 & 0.36 & 0.006 \\
\hline $\mathrm{V}_{30}\left(\mathrm{~cm}^{3}\right)$ & 8.70 & 19.03 & 0.33 & 0.01 \\
\hline $\mathrm{V}_{40}\left(\mathrm{~cm}^{3}\right)$ & 1.71 & 2.82 & 0.24 & 0.07 \\
\hline$V_{50}\left(\mathrm{~cm}^{3}\right)$ & 0.08 & 0.08 & 0.10 & 0.46 \\
\hline $\mathrm{D}_{2 \mathrm{~cm} 3}(\mathrm{~Gy} \mathrm{RBE})$ & 37.4 & 40.3 & 0.23 & 0.08 \\
\hline $\mathrm{D}_{1 \mathrm{~cm} 3}(\mathrm{~Gy} \mathrm{RBE})$ & 40.6 & 43.1 & 0.21 & 0.11 \\
\hline $\mathrm{D}_{\max }(\mathrm{Gy} \mathrm{RBE})$ & 51.0 & 51.5 & 0.05 & 0.69 \\
\hline
\end{tabular}

Table 4

Comparison of the actual incidence of gastrointestinal ulcer for each dosimetric parameter.

\begin{tabular}{llll}
\hline Parameter & Cutoff value $\left(\mathrm{cm}^{3}\right)$ & Actual incidence of ulcer $(\%)$ & $p$ \\
\hline $\mathrm{V}_{10}$ & $\geqslant 102$ & 51 & $<0.001$ \\
& $<102$ & 10 & \\
$\mathrm{~V}_{20}$ & $\geqslant 24$ & 42 & 0.008 \\
& $<24$ & 9 & \\
$\mathrm{~V}_{30}$ & $\geqslant 6$ & 34 & 0.015 \\
& $<6$ & 4 & \\
\hline
\end{tabular}

$51 \%$ vs. $10 \%\left(V_{10}, \geqslant 102 \mathrm{~cm}^{3}\right.$ or less $), 42 \%$ vs. $9 \%\left(V_{20}, \geqslant 24 \mathrm{~cm}^{3}\right.$ or less), $34 \%$ vs. $4 \%\left(V_{30}, \geqslant 6 \mathrm{~cm}^{3}\right.$ or less). The difference was statistically significant for each parameter.

\section{Discussion}

The incidence of severe upper GI ulcer or hemorrhage after CRT for pancreatic cancer was reported as approximately 10-30\% [1013]. In most studies, an endoscopic examination was performed in patients with symptoms, so that the incidence might have been underestimated [14]. Takatori et al. routinely conducted endoscopic examination and detected $49.4 \%$ radiation-induced ulcers after proton therapy including three grade $4 / 5$ GI toxicities during a 10 -month follow-up period [14]. In our series, we performed an endoscopic examination on a routine schedule after C-ion RT and detected $21 \%$ ulcers including grade $1 / 2$ in 11 (19\%) and grade 3 in only 1 (2\%). This is an extremely low incidence compared with 
other photon or proton therapies. This might prove that the dose constraint of $\mathrm{D}_{2 \mathrm{~cm} 3}<46 \mathrm{~Gy}$ (RBE) was appropriate.

Until now, few reports have been available on the relationship between GI volume and DVH for the treatment of pancreatic cancer. There are only two retrospective studies that demonstrated a dose-volume relationship regarding GI toxicity after CRT for pancreatic cancer $[12,13]$. Huang et al. reported that $37 \%$ had experienced grade 3 GI toxicity including 22\% grade 3 organic disorders: duodenal ulcer $(n=3)$, upper GI hemorrhage $(n=4)$, and small bowel obstruction $(n=3)$. The median time to GI toxicity was 1.3 months, and most of the toxicity occurred within 1 year after treatment. They concluded that $\mathrm{V}_{25}$ was the best predictor of GI toxicity [12]. Nakamura et al. reported that $20 \%$ of their CRT-treated pancreatic cancer patients experienced grade 3/4 GI ulcer or bleeding. Median time to severe GI toxicity was 4.1 months (range, $2.0-12.1$ ). They concluded that $\mathrm{V}_{50}$ was the best predictor for GI toxicity [13]. In our study, the median time was 2.1 months, and we found that GI toxicity significantly correlated with low-to-intermediate dose volume $\left(\mathrm{V}_{10-30}\right)$ as well as high dose volume $\left(D_{2 \mathrm{~cm} 3}\right)$, which agree with previous reports.

In the treatment of pancreatic cancer with C-ion RT, functional GI disorders such as anorexia, nausea, and vomiting have not been significant because the excellent dose concentration predominantly avoids the majority of the GI volume compared with conventional radiotherapy $[5,15]$. On the other hand, there might be the risk of organic disorders such as ulcer, bleeding, and perforation, because the high-intensity dose can affect a focal area of the GI mucosa. The highly conformal radiation field due to carbon-ion beams is strongly affected by various uncertainties such as organ motion and bowel gas [16]. To minimize the effect of these uncertainties, we routinely fix the patients with an immobilizing device and treat under a respiratory-gated system with a four-box field. Nevertheless, GI toxicity is a major dose-limiting factor because of the anatomical proximity of pancreatic cancer to the GI tract. Especially, respiratory organ motion is considered to be related closely to the location of ulcers, which appeared only in the stomach in our study. Four-dimensional CT analysis in pancreatic cancer treatment with C-ion RT revealed that organ motion in the gating phase is greatest toward the inferior side; the mean movement to the inferior side was determined to be $2.6-3.0 \mathrm{~mm}$ as contrasted to under $1.0 \mathrm{~mm}$ on the other side [17]. This means that the stomach, which is located on the superior marginal side of the PTV, may move into the high-dose area in PTV during irradiation time as a consequence of the respiratory motion. Meanwhile, the duodenum also moves mainly in an inferior direction, separating it from the PTV. This might be explained by the fact that ulcer locations mainly localized to the lower stomach as opposed to the duodenum, regardless of the tumor location.

These results from our study may not be applicable simply to other carbon-ion therapy facilities. Because there are several issues to be solved regarding the difference among carbon-ion therapy facilities such as RBE calculation, beam delivery system, beam arrangement, patient fixation, or respiratory-gated method. The appropriate dose constrains should be adapted carefully in each facility. However this study may provide a rough standard to treat pancreatic cancer with C-ion RT safely for other investigators. Furthermore, expanded use to proton therapy is difficult due to the difference of RBE and fractionation size.

There are some potential limitations of this study. First, different treatment regimens were included concerning the use of chemotherapy because of its retrospective nature. Information regarding patient-related parameters such as comorbidity, smoking, and alcohol use was unavailable. Especially, the adding of systemic agents to radiotherapy might have an impact on radiation-induced GI toxicity. However, the radiosensitizing effects observed with photon therapy are decreased with C-ion RT because the effectiveness of carbon ions is less cell-cycle-dependent [18]. A dose escalation trial of C-ion RT with concurrent gemcitabine for locally advanced pancreatic cancer showed only independent toxicities [5]. Hence, we think the different regimen of systemic agents had an insignificant effect on GI ulcer. Second, the follow-up period might be too short to evaluate late toxicity. The survival time of inoperable pancreatic cancer is short, only $10-16$ months $[2,19,20]$, making it difficult to evaluate any long-term effect of GI toxicity. Although previous reports explained that the median time to GI toxicity was 2-4 months and most toxicity occurred within 12 months [12,13,21], a longer follow-up would be needed. Third, the DVH parameters investigated in this study are estimated based on the treatment planning scan and may not reflect the actual dose received. Intra- and interfractional organ motion can affect the DVH parameters. Actually, the DVH parameters in one grade 3 patient were low compared with those in the 11 grade $1 / 2$ ulcer patients. Our thresholds of DVH parameters might not reflect the risk of severe GI toxicity accurately. Thus, further investigation will be needed to reveal the dose constraint of the GI tract in the treatment of C-ion RT. Our results will help others prevent severe GI toxicity from occurring by foreseeing low-grade GI toxicity using the organspecific dosimetric parameters determined here. Also, these results can provide a basis for scanning beam-delivered C-ion RT in a doseescalation clinical trial.

In conclusion, $\mathrm{C}$-ion RT for pancreatic cancer under a GI dose constraint of $\mathrm{D}_{2 \mathrm{~cm} 3}<46 \mathrm{~Gy}$ (RBE) decreased the risk of GI ulcer. To further minimize the risk of GI ulcer, a low-to-intermediate dose volume $\left(\mathrm{V}_{10-30}\right)$ should be also reduced.

\section{Conflict of interest}

None.

\section{References}

[1] Chauffert B, Mornex F, Bonnetain F, et al. Phase III trial comparing intensive induction chemoradiotherapy (60 Gy, infusional 5-FU and intermittent cisplatin) followed by maintenance gemcitabine with gemcitabine alone for locally advanced unresectable pancreatic cancer. Definitive results of the 2000-01 FFCD/SFRO study. Ann Oncol 2008;19:1592-9.

[2] Loehrer Sr PJ, Feng Y, Cardenes H, et al. Gemcitabine alone versus gemcitabine plus radiotherapy in patients with locally advanced pancreatic cancer: an Eastern Cooperative Oncology Group trial. J Clin Oncol 2011;29:4105-12.

[3] Hammel P, Huguet F, Laethem DJ. Comparison of chemoradiotherapy (CRT) and chemotherapy (CT) in patients with a locally advanced pancreatic cancer (LAPC) controlled after 4 months of gemcitabine with or without erlotinib: final results of the international phase III LAP 07 study. J Clin Oncol 2013. abstr LBA 4003.

[4] Oonishi K, Cui X, Hirakawa H, et al. Different effects of carbon ion beams and Xrays on clonogenic survival and DNA repair in human pancreatic cancer stemlike cells. Radiother Oncol 2012;105:258-65.

[5] Shinoto M, Yamada S, Terashima K, et al. Carbon ion radiation therapy with concurrent gemcitabine for patients with locally advanced pancreatic cancer. Int J Radiat Oncol Biol Phys 2015.

[6] Kanai T, Endo M, Minohara S, et al. Biophysical characteristics of HIMAC clinical irradiation system for heavy-ion radiation therapy. Int J Radiat Oncol Biol Phys 1999;44:201-10.

[7] Kamada T, Tsujii H, Blakely EA, et al. Carbon ion radiotherapy in Japan: an assessment of 20 years of clinical experience. Lancet Oncol 2015;16:e93-e100.

[8] DeLuca PM. Prescribing, recording, and reporting proton-beam therapy (supplement to ICRU report 78): International Commission on Radiation Units and Measurements, 2007.

[9] Society JP. Classification of Pancreatic carcinoma (English edition). 3rd ed, 2011. Kanehara, Tokyo.

[10] Shibuya K, Oya N, Fujii T, et al. Phase II study of radiation therapy combined with weekly low-dose gemcitabine for locally advanced, unresectable pancreatic cancer. Am J Clin Oncol 2011;34:115-9.

[11] de Lange SM, van Groeningen CJ, Meijer OW, et al. Gemcitabine-radiotherapy in patients with locally advanced pancreatic cancer. Eur J Cancer 2002;38:1212-7.

[12] Huang J, Robertson JM, Ye H, Margolis J, Nadeau L, Yan D. Dose-volume analysis of predictors for gastrointestinal toxicity after concurrent full-dose gemcitabine and radiotherapy for locally advanced pancreatic adenocarcinoma. Int J Radiat Oncol Biol Phys 2012;83:1120-5. 
[13] Nakamura A, Shibuya K, Matsuo Y, et al. Analysis of dosimetric parameters associated with acute gastrointestinal toxicity and upper gastrointestinal bleeding in locally advanced pancreatic cancer patients treated with gemcitabine-based concurrent chemoradiotherapy. Int J Radiat Oncol Biol Phys 2012;84:369-75.

[14] Takatori K, Terashima K, Yoshida R, et al. Upper gastrointestinal complications associated with gemcitabine-concurrent proton radiotherapy for inoperable pancreatic cancer. J Gastroenterol 2013.

[15] Shinoto M, Yamada S, Yasuda S, et al. Phase 1 trial of preoperative, shortcourse carbon-ion radiotherapy for patients with resectable pancreatic cancer. Cancer 2013;119:45-51.

[16] Kumagai M, Hara R, Mori S, et al. Impact of intrafractional bowel gas movement on carbon ion beam dose distribution in pancreatic radiotherapy. Int J Radiat Oncol Biol Phys 2009;73:1276-81.
17] Mori S, Hara R, Yanagi T, et al. Four-dimensional measurement of intrafractional respiratory motion of pancreatic tumors using a 256 multislice CT scanner. Radiother Oncol 2009;92:231-7.

[18] Schlaich F, Brons S, Haberer T, Debus J, Combs SE, Weber KJ. Comparison of the effects of photon versus carbon ion irradiation when combined with chemotherapy in vitro. Radiat Oncol 2013;8:260.

[19] Ishii H, Furuse J, Boku N, et al. Phase II study of gemcitabine chemotherapy alone for locally advanced pancreatic carcinoma: JCOG0506. Jpn J Clin Oncol 2010;40:573-9.

[20] Ikeda M, Ioka T, Ito Y, et al. A multicenter phase II trial of S-1 with concurrent radiation therapy for locally advanced pancreatic cancer. Int J Radiat Oncol Bio Phys 2013;85:163-9.

[21] Coia LR, Myerson RJ, Tepper JE. Late effects of radiation therapy on the gastrointestinal tract. Int J Radiat Oncol Biol Phys 1995;31:1213-36. 